\title{
Strategic trends in the vaccine market
}

\author{
Hedwig Kresse and Mansi Shah
}

The vaccine market has grown rapidly in the past 5 years. Reported sales of vaccines globally from the five largest companies in the field - Sanofi Pasteur, GlaxoSmithKline, Merck \& Co, Pfizer and Novartis - which account for an estimated $80 \%$ of the total market $^{1}$, grew from US $\$ 7.1$ billion in 2004 to over $\$ 20$ billion in 2009 .

This strong performance has been driven by the launch and rapid uptake of novel, high-price vaccines, as well as by pandemic influenza vaccine sales. However, cost-containment, increasing competition in established areas such as influenza, a lack of innovative late-stage pipeline candidates and a strong anti-vaccination lobby are key challenges to the continuing growth of this sector.

\section{The changing vaccine market}

Prior to 2000, pharmaceutical and biotechnology companies generally regarded vaccines as a market with limited revenue opportunities. However, a growing awareness of the benefits of vaccination, an improved understanding of pathogen biology and the human immune system and more favourable legislation regarding vaccine injury compensation have made

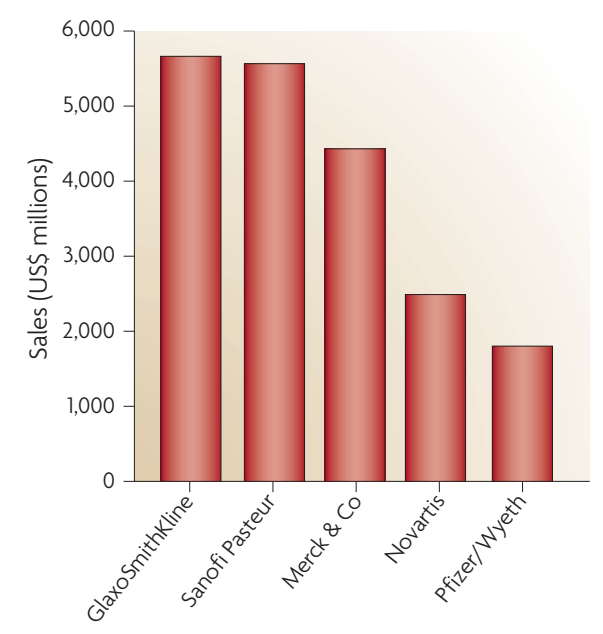

Figure 1| Vaccine sales of the five largest companies in the field in 2009. Source: company reports. vaccine development more attractive. Furthermore, novel vaccine production and adjuvant technologies have aided the development of vaccines for new indications with a high unmet need.

The commercial potential of vaccines has been proven by the success of novel, high-price products such as the pneumococcal conjugate vaccine Prevnar (Wyeth, now Pfizer) and the quadrivalent human papillomavirus vaccine Gardasil (Merck \& Co) (TABLE 1). As large pharmaceutical companies are facing major revenue losses due to competition from generic versions of branded drugs, vaccines - for which the barrier to the entry of a generic version is much higher - are increasingly becoming commercially attractive.

\section{Current market}

The vaccine market is dominated by five companies - Sanofi Pasteur, Merck \& Co, GlaxoSmithKline, Pfizer (following the 2009 acquisition of Wyeth) and Novartis (FIG. 1). Established vaccine classes such as influenza, hepatitis and paediatric vaccines account for the largest share of global vaccine sales. The outbreak of the H1N1 pandemic in 2009 led to substantial revenue increases for all major influenza vaccine players, with GlaxoSmithKline, Sanofi Pasteur and Novartis reporting combined pandemic influenza sales of $\$ 6.4$ billion in 2009 and the first half of 2010. However, these high gains are likely to be short-lived, given that stockpiling of pandemic influenza vaccines has considerably subsided in 2010 .

On a product level, several innovative vaccines stand out as particularly successful. Prevnar and Gardasil have both achieved blockbuster status with global sales of over \$1.5 billion each in 2009 . Moreover, the quadrivalent conjugate meningitis vaccine Menactra (Sanofi Pasteur), the infant combination vaccine Pentacel (Sanofi Pasteur) and the rotavirus vaccine RotaTeq (Merck \& Co) achieved global sales in excess of $\$ 500$ million each in 2009.
‘Baba Papa Lounger' by Haldane Martin.

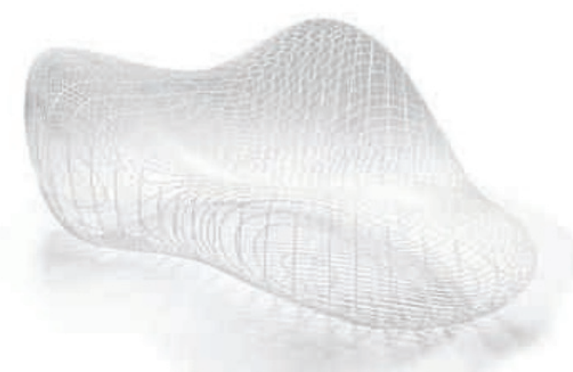

Entry of new pharmaceutical companies Driven by its increasing commercial potential, the vaccines sector has undergone significant merger and acquisition activity in the past 5 years, with several large pharmaceutical companies entering the field or strengthening their position (TIMELINE). However, their commercial outlook in this sector varies significantly.

Pfizer has built a vaccine portfolio through the acquisitions of PowderMed (2006), Coley (2007) and, most importantly, Wyeth (2009). Through the deal with Wyeth, Pfizer gained access to Prevnar, an attractive vaccine pipeline and an experienced team. In 2007, AstraZeneca also entered the vaccine sector through the acquisition of MedImmune, which markets the intranasal influenza vaccine FluMist. However, although AstraZeneca gained windfall revenues owing to the 2009/10 H1N1 pandemic, FluMist's overall commercial performance has been disappointing, and we expect AstraZeneca to remain a niche company in the vaccine sector overall. Similarly, Abbott obtained an influenza vaccine portfolio through acquiring Solvay's Pharma business in 2009. However, owing to portfolio considerations, as well as a lack of competitiveness in this sector, Abbott attempted to sell its vaccine business shortly after the acquisition. Failing to attract a buyer, the company announced in September 2010 that it would cease most vaccine research and development activities and will mainly continue to produce and sell its seasonal influenza vaccine Influvac.

The latest entrant is Johnson \& Johnson, which agreed to fully acquire Crucell for $\$ 2.4$ billion in October 2010. We believe that Crucell's attractive vaccine portfolio and pipeline will further reinforce Johnson \& Johnson's already strong position in the infectious diseases sector. Overall, these developments illustrate the importance of expertise and a well-planned strategy in achieving success in the vaccine sector, which differs significantly from other sectors of the pharmaceutical industry with regard to clinical development, production, marketing and distribution. 


\section{VACCINE MARKET | MARKET INDICATORS}

\section{- New targets and emerging markets}

Facing increasingly saturated markets for basic vaccines in North America and in Europe, companies are progressively looking to expand into other regions. Emerging and developing countries represent a promising commercial opportunity for vaccine makers owing to their higher disease burden and, consequently, a large unmet medical need for even basic vaccines. Local governments are also increasing their efforts to improve vaccination rates and assure supply, while growing middle classes and increasing health-care spending are creating a significant private sector opportunity. This is reflected in a multitude of deals and alliances between the major vaccine developers and domestic vaccine makers in countries such as India, China and Brazil (see Supplementary information S1 (table)).

New high-value targets also hold further opportunities for vaccine developers. With regard to prophylactic vaccines, meningitis B is widely thought of as the next near-term blockbuster opportunity, with two candidate vaccines being developed by Pfizer and Novartis in late-stage clinical trials. Developing vaccines against infections of nosocomial pathogens such as Staphylococcus aureus or Pseudomonas aeruginosa is also attractive, as increasing bacterial resistance is limiting the efficacy of current antibiotics. Vaccines against tropical diseases such as Japanese encephalitis or dengue fever offer a dual, tiered-priced market opportunity for both selected target populations (for example, travellers and army recruits) in the developed world, as well as a wider target population in endemic countries.

In addition, the approval by the US Food and Drug Administration in April 2010 of the first therapeutic cancer vaccine

\begin{tabular}{|c|c|c|c|}
\hline Vaccine & Company & Indication & $\begin{array}{l}2009 \text { reported } \\
\text { sales (US\$ million) }\end{array}$ \\
\hline Prevnar & Pfizer & Pneumococcal disease & 1,826 \\
\hline Gardasil & Merck \& Co & Human papilloma virus & 1,667 \\
\hline Menactra & Sanofi Pasteur & $\begin{array}{l}\text { Neisseria meningitidis serogroups A, C, Y } \\
\text { and W-135 }\end{array}$ & 619 \\
\hline RotaTeq & Merck \& Co & Rotavirus infection & 563 \\
\hline Pentacel & Sanofi Pasteur & $\begin{array}{l}\text { Diphtheria, tetanus, pertussis, poliomyelitis, } \\
\text { invasive Haemophilus influenzae type b }\end{array}$ & 507 \\
\hline
\end{tabular}

- sipuleucel-T (Provenge; Dendreon) for the treatment of prostate cancer has reinvigorated interest in therapeutic vaccines for a wide range of indications across several therapeutic areas, including Alzheimer's disease, substance dependence, hepatitis C and HIV. For more established prophylactic vaccine indications such as influenza or certain childhood vaccines, novel technologies such as improved adjuvants and needle-free or mucosal delivery, as well as more rapid and more flexible production methods, offer scope for product differentiation and improvement.

\section{Future of vaccine development} In the light of increasing competition, saturation of key vaccine sectors and a lack of innovative late-stage vaccine candidates, maintaining the strong growth of the past decade will be challenging. As high-revenue vaccines such as Prevnar or Gardasil reach maturity, the development of new blockbusters is hindered by scientific challenges: marketed vaccines already exist against most easy-to-address targets, leaving only complex pathogens such as hepatitis $C$ and HIV. Furthermore, following a period of rapid introduction, reimbursement and uptake for high-price vaccines such as Prevnar, Gardasil, Menactra and RotaTeq, health-care providers in North America and in Europe have started to implement stricter cost-containment measures in the wake of the economic downturn. Looking forward, this will make it harder for pharmaceutical companies to recover the increasing development costs for more complex vaccines.

Other factors limiting vaccine uptake include the widespread lack of disease awareness and a public apprehension of vaccination and new vaccine-related technologies. Owing to the prophylactic nature of most marketed and pipeline vaccines and their consequent use in healthy people, an excellent safety and tolerability profile for new candidates will remain paramount in vaccine development.

Hedwig Kresse and Mansi Shah are analysts at Datamonitor Healthcare, 119 Farringdon Road, London EC1R 3DA, UK e-mail: hkresse@datamonitor.com doi: $10.1038 /$ nrd3306

1. IMS Health. IMS Health MIDAS sales data, 2006-2009.

Competing interests statement

The authors declare no competing financial interests.

SUPPLEMENTARY INFORMATION See online article: $\underline{\mathrm{S}}$ (table)

ALL LINKS ARE ACTIVE IN THE ONLINE PDF

Timeline | Market entry of large pharmaceutical companies

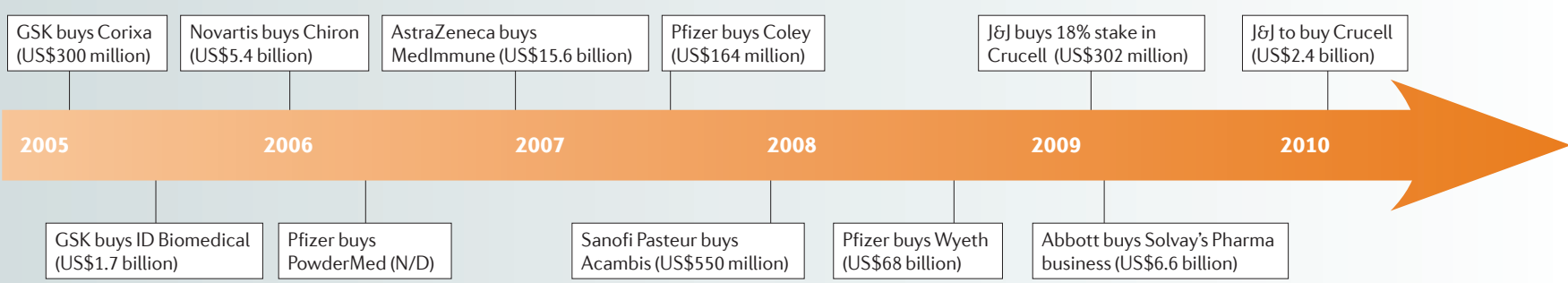

JEJ, Johnson \& Johnson; N/D, not disclosed. 